\title{
The Design and Analysis Based on the Fresnel Condenser Lens
}

\author{
Heli Wu ${ }^{1, \text { a }}$, Fan Yang ${ }^{2, \mathrm{~b}}$, Lihong Zhou ${ }^{3, \mathrm{c}}$ \\ ${ }^{1,2,3}$ City College, Wuhan University of Science and Technology, Wuhan, Hubei 430083, P. R. China \\ ayunflyen@163.com, blihongzhou2004@163.com,
}

Keywords: Fresnel Lens; Optical simulation; Refractive index

Abstract. From the study of the CPV Important components based on the Condenser design of Fresnel lens, through extracting the solar spectral data, modeling condenser materials, simulating condenser of sunlight by optical simulation software, and also simulation light path of sunlight, this thesis will complete the structure's design and analysis of the Fresnel condenser lens.

\section{Introduction}

The Fresnel Lens was invented by the French physicist Augustin Jean Fresnel in 1822[1], which is built by the prism of the multiple coaxial or parallel arrangement forming a sequence of discrete surface to replace the general continuous spherical lens. Therefore, Fresnel lens is simple structure, easy manufacturing, lighter and thinner than the general lens from the weight and volume, and to obtain larger aperture and focal length ratio in design.

The control index of Fresnel lens is mainly on the optical efficiency, energy distribution uniformity on image plane、 dispersion, the spot size of the condensation and the match of the CPV cells, etc.

\section{The general design method of Fresnel lens}

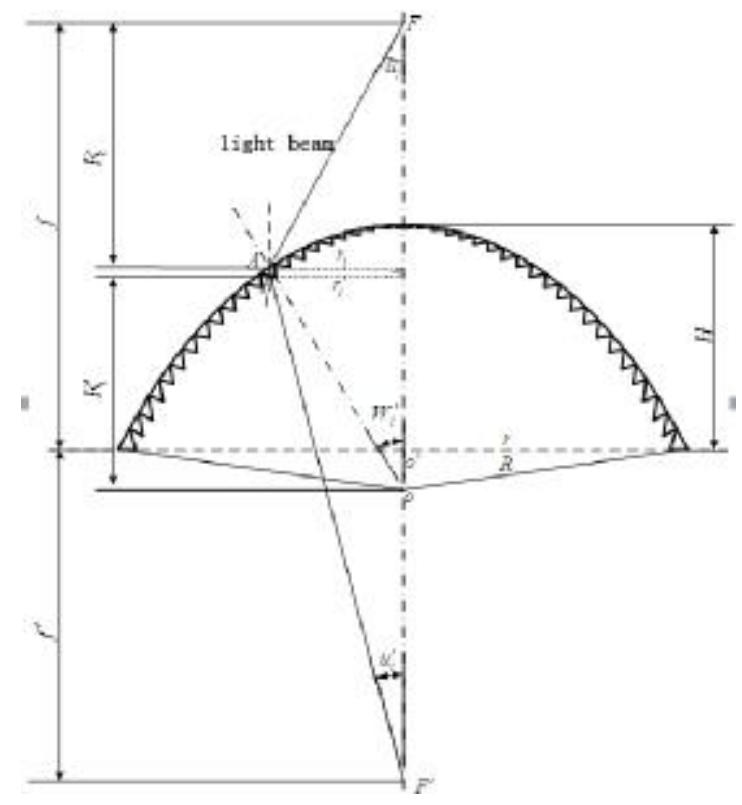

Fig.1 Fresnel lens schematic diagram

Assuming a light source is at the point of $\mathrm{F}$, and its beam starts from $\mathrm{F}$, then it will focus on the other side of the lens by reflecting from the medium. And a beam of light passes from the edge of the lens through the refraction from $\mathrm{A}$ to $\mathrm{B}$, then it refracts in the focus point $F^{\prime} . O$ and $O^{\prime}$ are the center of the theory of the arc cross section and the center of the arc cross section[2]。 


$$
\theta_{i}=W_{i}^{\prime}+\tan ^{-1} \frac{\left(\begin{array}{l}
\sin W_{i}^{\prime} \cdot \sqrt{N^{2}-\sin ^{2}\left(u_{i}+W_{i}^{\prime}\right)}- \\
\cos W_{i}^{\prime} \sin \left(u_{i}+W_{i}^{\prime}\right)-\sin u_{i}^{\prime}
\end{array}\right)}{\left(\begin{array}{l}
\cos u_{i}^{\prime}-\cos W_{i}^{\prime} \cdot \sqrt{N^{2}-\sin ^{2}\left(u_{i}+W_{i}^{\prime}\right)} \\
-\sin W_{i}^{\prime} \sin \left(u_{i}+W_{i}^{\prime}\right)
\end{array}\right)}
$$

Eq.1 is the general design for the Fresnel condenser lens, and by limiting the parameters we can obtain a design formula of the different structural lens. The following is the design formulas for Fresnel condensing lenses under specified conditions:

When the prism faces inward, the parallel light is incident from the surface of the light, $f=\infty$, $u_{i}=0^{\circ}$ and Eq. 1 will become the following:

$$
\theta_{i}=W_{i}^{\prime}+\tan ^{-1} \frac{\left(\begin{array}{l}
\sin W_{i}^{\prime} \cdot \sqrt{N^{2}-\sin ^{2} W_{i}^{\prime}}- \\
\cos W_{i}^{\prime} \sin W_{i}^{\prime}-\sin u_{i}^{\prime}
\end{array}\right)}{\left(\begin{array}{l}
\cos u_{i}^{\prime}-\sin ^{2} W_{i}^{\prime}- \\
\cos W_{i}^{\prime} \cdot \sqrt{N^{2}-\sin ^{2} W_{i}^{\prime}}
\end{array}\right)}
$$

When the edge faces outward, the incidence of the parallel light is from the edge, $f^{\prime}=\infty$, $u_{i}^{\prime}=0^{\circ}$, then the Eq.1 turns into:

$$
\theta_{i}=W_{i}^{\prime}+\tan ^{-1} \frac{\left(\begin{array}{l}
\sin W_{i}^{\prime} \cdot \sqrt{N^{2}-\sin ^{2}\left(u_{i}+W_{i}^{\prime}\right)}- \\
\cos W_{i}^{\prime} \sin \left(u_{i}+W_{i}^{\prime}\right)
\end{array}\right)}{\left(\begin{array}{l}
1-\cos W_{i}^{\prime} \cdot \sqrt{N^{2}-\sin ^{2}\left(u_{i}+W_{i}^{\prime}\right)} \\
-\sin W_{i}^{\prime} \sin \left(u_{i}+W_{i}^{\prime}\right)
\end{array}\right)}
$$

Similarly, when the edge is inward, the incidence of the parallel light is from shiny side, $f=\infty$, $u_{i}=0^{\circ}$, Eq 2 becomes:

$$
\theta_{i}=\tan ^{-1}\left(\frac{\sin u_{i}^{\prime}}{N-\cos u_{i}^{\prime}}\right)
$$

When the edge faces outward, the incidence of the parallel light is from the edge, $f^{\prime}=\infty, \quad u_{i}^{\prime}=0^{\circ}$, then Eq. 2 is transformed into:

$$
\theta_{i}=\tan ^{-1}\left(\frac{\sin u_{i}}{\sqrt{N^{2}-\sin ^{2} u_{i}}-1}\right)
$$

\section{Stimulation of the solar spectrum}

Sunlight is due to the intense radiation produced by the the sun thermonuclear fusion reaction. It is the energy which life beings in the earth rely on and ensures that the whole world is a blaze of 
color, rich and colorful.The solar spectrum mainly contains three parts: infrared ray, visible light, and ultraviolet light, the spectrum of which ranges from $300 \mathrm{~nm}$ to $3000 \mathrm{~nm}$. The light near the red light contains a larger heat energy proportionally, and the energy of ultraviolet is smaller.

Because the NASA's solar data of simulating the solar spectrum data provides fewer data, this thesis is to obtain more spectrum proportion according to interpolation method to make the solar spectrum simulation more exact.

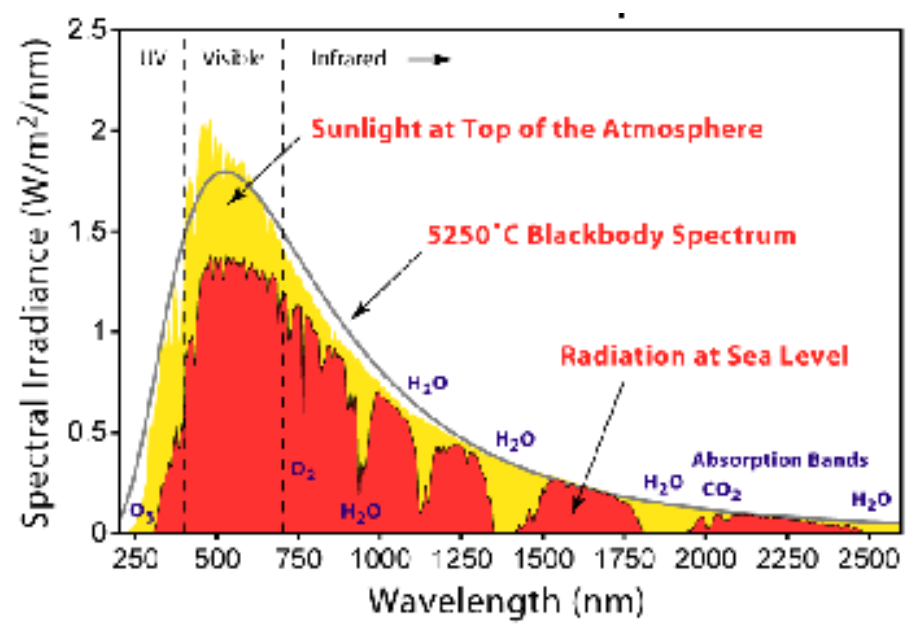

Fig.2 Solar Radiation Spectrum[3]

The comparison of the data curves of simulating sunlight and the actual sunlight

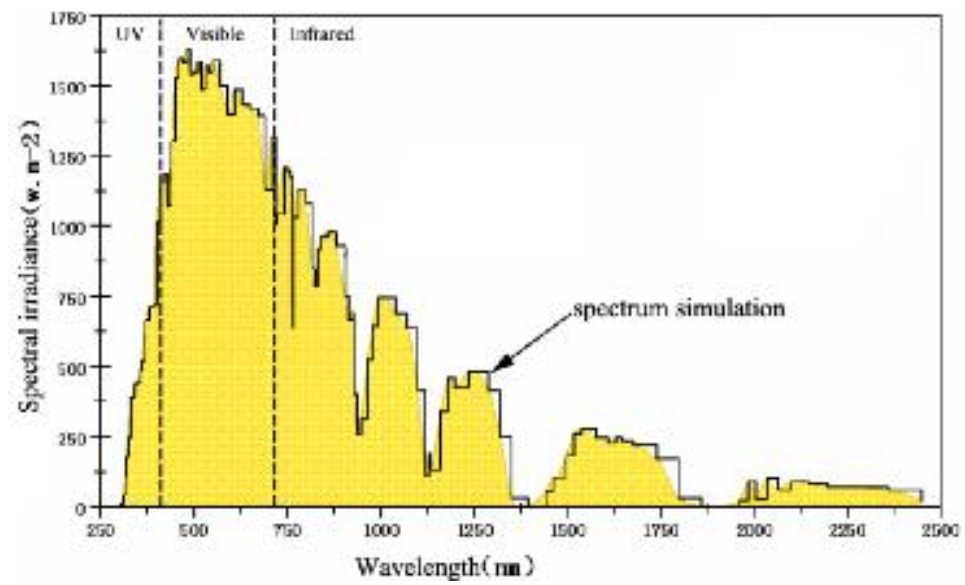

Fig.3 Simulating the Solar Spectrum

\section{Performance simulation of the material of the optical device}

There is a variety of material to make Fresnel lens, the best one is the Acrylic material which is possessed of superior strength and toughness and good light transmittance.PMMA material as one of the Acrylic is one type of important thermoplastic polymer developed earlier. Its better transparency, chemical stability and weather resistance, easy dyeing, easy processing and beautiful appearance make it widely used in aerospace, automotive, electronics, medical, chemical, building materials, sanitary ware and advertising signs, etc.

Because optical material BK7 is also widely applied, this paper will be compare it with PMMA material accordingly. 


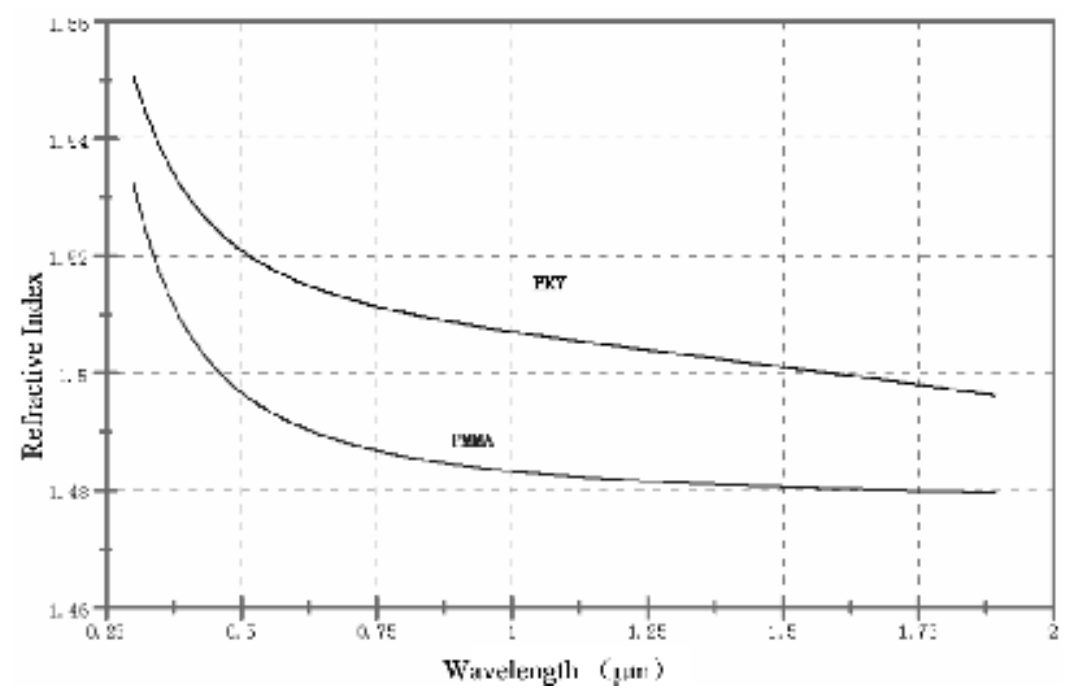

Fig.4 The Refractive Index of BK7 and PMMA for Different Wavelength of Light

\section{The optical simulation of Fresnel lens's Tracepro}

TracePro, as the next generation software to analyze the deviation of light, needs to be able to analyze the rays effectively and accurately. To achieve these goals, TracePro has the following functions: the ability to handle complex geometry, to define and track millions of light rays; to display graphical, visual operating, to provide databases of 3D solid model; to Import and export the data format of mainstream CAD software and designing software of lens.

TracePro is easy to use with only five steps to use: to build geometric models; to set up optical materials; to define the source parameters; to track the ray of light; and to analyze the simulation results.

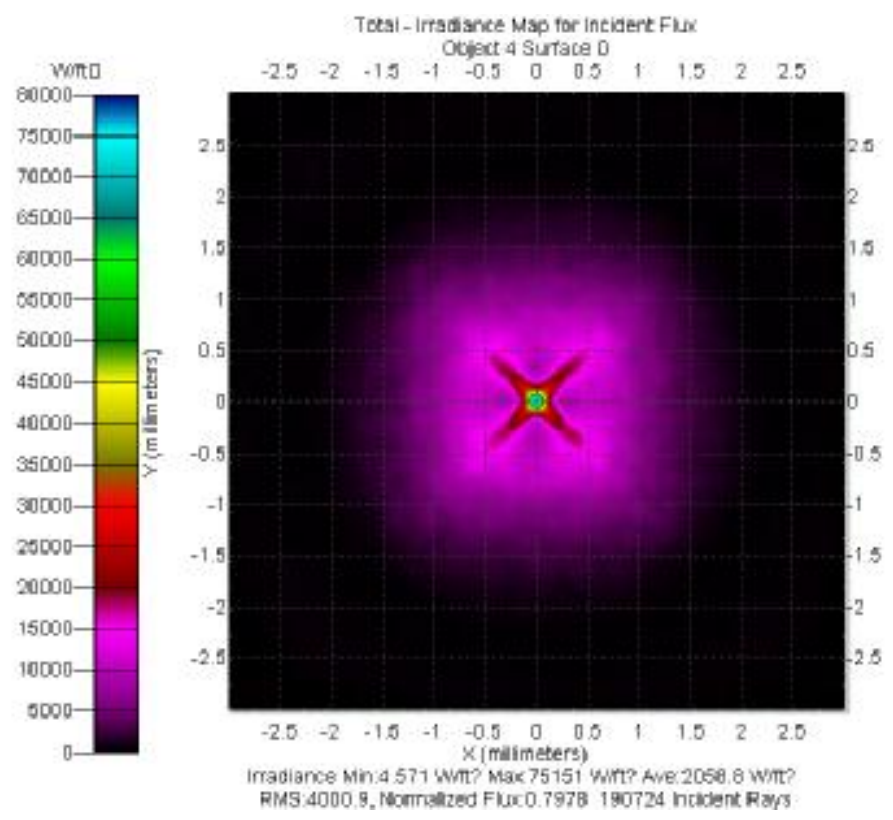

Figs.5 The Spot is in front of the Focus $\mathrm{f}=85$ 

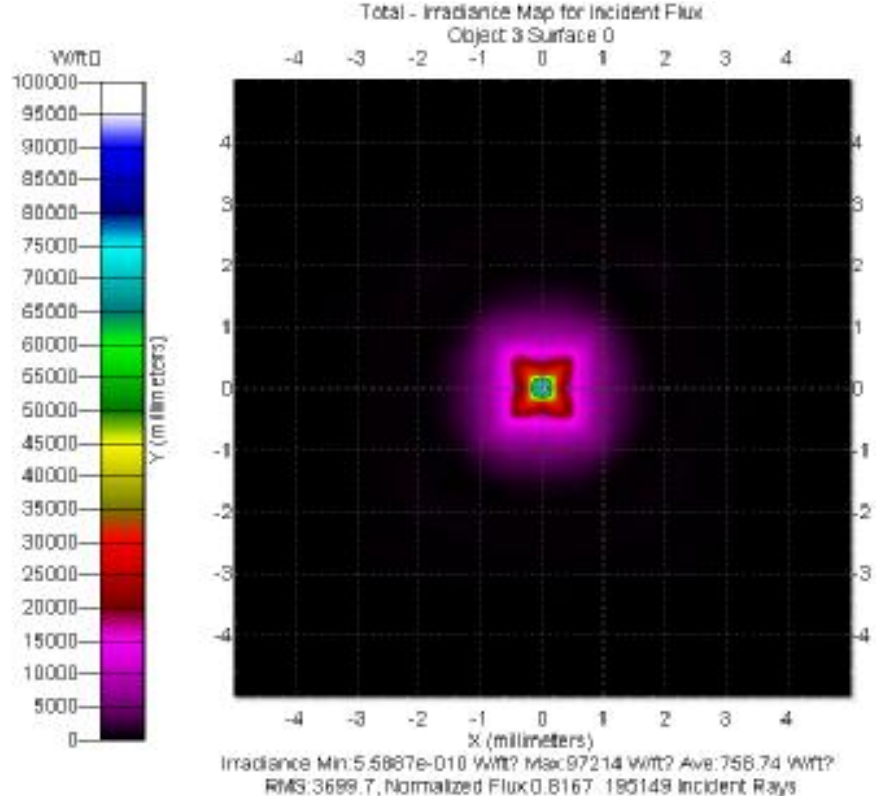

Figs. 6 The Spot is in the Focused Point $\mathrm{f}=90$

Tetai - Irradiance Map for intidert Flux
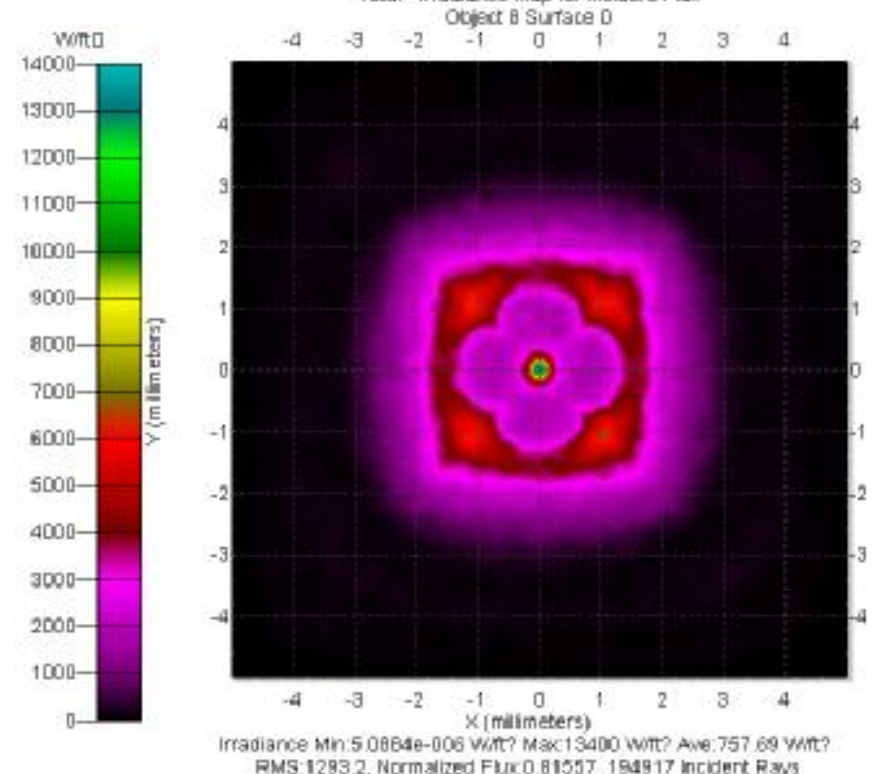

Figs.7 The Spot is in the Back Focus $\mathrm{f}=95$

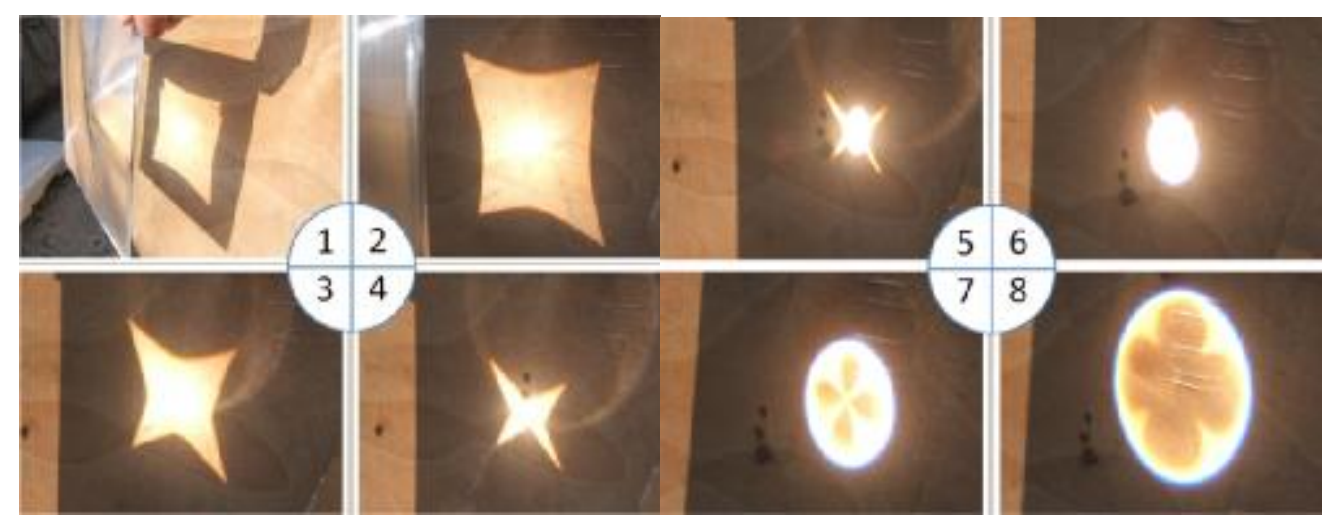

Fig.8 Experiment Picture of Focusing Spot Size of the Fresnel Lens

\section{Conclusion}

The design and analysis of Fresnel Condenser lens provide the theoretical basis of usage of clean 
solar energy, and through the simulation of the solar spectrum energy and the reconstruction simulation of the actual experiment by the design of Tracepro software. Theory provides the support for the practice, and practice verifies the theoretical simulation. It provides a feasible scheme for the usage and design of the solar energy concentrated power.

\section{Reference}

[1]Shensheng Li. Solar Energy. Beijing: Beijing People's Education Press. 1988

[2]Peng Li, Heli Wu, Peihuan Yang. The Common Design Method and Efficiency Analysis of Fresnel Condenser lens. Wu Han: Journal of Wuhan University of Technology, 2010,32(6): 62-66 [3] http://upload.wikimedia.org/wikipedia/commons/4/4c/Solar_Spectrum.png 